\title{
FEATURE EXTRACTION OF DORSAL HAND VEIN FOR AUTHENTICATION SYSTEM
}

\author{
S.Karthika Rajaratna, P.Keerthana, S.Laxmi Priya \\ National Engineering College,Kovilpatti-628 503. \\ Email: rathna1994@yahoo.com
}

\begin{abstract}
The Dorsal hand vein identification system is used for identifying the vein patterns in a person's dorsal hand. Vein pattern identification uses an infrared light source to scan for haemoglobin within the blood. Once the user's hand is kept over a sensing device, a near infrared light from the sensing device maps the position of the veins. Deoxygenated hemoglobin flowing in the veins absorb these infrared rays and show up on the map as black lines, whereas the remaining portion of hand structure shows up as white. The dorsal side of hands and palms have more complex vascular patterns than fingers and provide a lot of distinct features for pattern matching and authentication. Images suffer from quality degradation due to transmission of limited range of light, low contrast and blurred image due to quality of light and diminishing color. The performance of an image filtering system depends on its ability to detect the presence of noisy pixels in the image. Different filtering techniques are available for improving the images, as the filtering methods remove noise from the image, contrast enhancement tends to enhance the contrast of the image and segmentation method to extract the foreground image from the background.

This technology is a good alternative to biometric systems that require physical contact because it extracts the vein pattern, the hand is not in contact with the device instead hand is just easily stretched and the capturing of vein pattern is completed. Due to non-contact, it is hygienic. Since the system has got the three features live body, internal features and non-contact, there is no forgery, and no misuse by evildoers, thus it grasps high security status and can be used at high level security places.
\end{abstract}

